\title{
Optimal Pair of Two Linear Varieties
}

\author{
Armando Gonçalves ${ }^{1}$, M. A. Facas Vicente ${ }^{* 1}{ }^{2}$ And José Vitória ${ }^{1}$
}

\begin{abstract}
The optimal pair of two linear varieties is considered as a best approximation problem, namely the distance between a point and the difference set of two linear varieties. The Gram determinant allows to get the optimal pair in closed form.
\end{abstract}

\section{INTRODUCTION}

In this paper we deal with the problem of finding the the optimal points of two linear varieties in a finite dimensional real linear space.

The distance between two linear varieties has been dealt with in several papers: 2], [3] and [5]. In [5], only the distance is considered and the points that realize the distance are not exhibited. In 2 the best approximation points are found.

In [2] projecting equations were called into play; in [3] the difference set of two closed convex sets in $\mathbb{R}^{m}$ was considered.

In this paper, we formulate the problem as suggested by [3, page 196] and we use Gram theory [9, page 74], 44, page 65] to solve it.

We use results on existence and uniqueness of optimal points by considering a least norm problem of the difference set of two closed convex sets in $\mathbb{R}^{m}[3$. For the concepts, results and motivation on the study of the distance between convex sets see 3 .

We endow the space $\mathbb{R}^{m}$ with the usual inner product $\bullet$ :

$$
\vec{p} \bullet \vec{q}:=p_{1} q_{1}+p_{2} q_{2}+\cdots p_{m} q_{m},
$$

where $\vec{p}=\left[\begin{array}{llll}p_{1} & p_{2} & \cdots & p_{m}\end{array}\right]^{T}$ and $\vec{q}=\left[\begin{array}{llll}q_{1} & q_{2} & \cdots & q_{m}\end{array}\right]^{T}$ and with the Euclidean norm

$$
\|\vec{p}\|=\sqrt{\vec{p} \bullet \vec{p}}
$$

* Corresponding author.

2010 Mathematics Subject Classification. 41A50, 41A52, 51M16, 51N20.

Key words and phrases. linear varieties, best approximation pair, approximation theory.

${ }^{1}$ Department of Mathematics, University of Coimbra, Apartado 3008, EC Santa Cruz, 3001-501 Coimbra, Portugal. E-mails: adsg@mat.uc.pt (Armando Gonçalves), vicente@mat.uc.pt (M. A. Facas Vicente) and jvitoria@mat.uc.pt (José Vitória).

${ }^{2}$ Supported by Instituto de Engenharia de Sistemas e Computadores-Coimbra, Rua Antero de Quental, 199, 3000-033 Coimbra, Portugal. 


\section{The Result}

We are looking for the optimal pair of two linear varieties $V_{\vec{b}}$ and $V_{\vec{c}}$ in $\mathbb{R}^{m}$ defined by

$$
V_{\vec{b}}:=\left\{\vec{b}+B \vec{u}: \vec{u} \in \mathbb{R}^{l_{1}}\right\} \text { and } V_{\vec{c}}:=\left\{\vec{c}-C \vec{v}: \vec{v} \in \mathbb{R}^{l_{2}}\right\},
$$

with $\vec{b}$ and $\vec{c}$ given vectors in $\mathbb{R}^{m}$.

Following [3, page 196] the distance $d\left(V_{\vec{b}}, V_{\vec{c}}\right)$ between the two linear varieties $V_{\vec{b}}$ and $V_{\vec{c}}$ is obtained through the minimization of $\|A \vec{x}-\vec{d}\|$ where:

$\vec{d}=\vec{c}-\vec{b} ; A=[B C]$ is a real $m \times\left(l_{1}+l_{2}\right)$ matrix; and $\vec{x}=\left[\vec{u}^{T} \vec{v}^{T}\right]^{T} \in \mathbb{R}^{l_{1}+l_{2}}$.

So the distance $d\left(V_{\vec{b}}, V_{\vec{c}}\right)$ between the varieties $V_{\vec{b}}$ and $V_{\vec{c}}$, may be studied using the shortest distance between a point $\vec{d}$ and the subspace Range $(A)$, the column space of matrix $A$.

Besides getting the distance $d\left(V_{\vec{b}}, V_{\vec{c}}\right)$, we also find the points $\vec{b}^{*} \in V_{\vec{b}}$ and $\vec{c}^{*} \in V_{\vec{c}}$ such that $d\left(V_{\vec{b}}, V_{\vec{c}}\right)=\left\|\vec{b}^{*}-\vec{c}^{*}\right\|$, that is to say $\vec{b}^{*}$ and $\vec{c}^{*}$ are the best approximation points.

In this new setting, and using the Euclidean norm, Gram theory 9] can play an important role.

Some definitions are needed.

Definition 2.1. The optimal pair of two linear varieties $\mathcal{A}$ and $\mathcal{B}$ is the pair $\left(\vec{a}^{*}, \vec{b}^{*}\right) \in$ $\mathcal{A} \times \mathcal{B}$ satisfying $d(\mathcal{A}, \mathcal{B})=\left\|\vec{a}^{*}-\vec{b}^{*}\right\|$, where $d(\mathcal{A}, \mathcal{B})$, the distance between $\mathcal{A}$ and $\mathcal{B}$, is defined as

$$
d(\mathcal{A}, \mathcal{B})=\inf \{\|\vec{a}-\vec{b}\|: \vec{a} \in \mathcal{A}, \vec{b} \in \mathcal{B}\} .
$$

Definition 2.2. Let $y_{1}, y_{2}, \ldots, y_{n}$ be elements of $\mathbb{R}^{m}$. The $n \times n$ matrix

$$
G\left(y_{1}, y_{2}, \ldots, y_{n}\right)=\left[\begin{array}{cccc}
y_{1} \bullet y_{1} & y_{1} \bullet y_{2} & \cdots & y_{1} \bullet y_{n} \\
y_{2} \bullet y_{1} & y_{2} \bullet y_{2} & \cdots & y_{2} \bullet y_{n} \\
\vdots & \vdots & \vdots & \vdots \\
y_{n} \bullet y_{1} & y_{n} \bullet y_{2} & \cdots & y_{n} \bullet y_{n}
\end{array}\right]
$$

is called the Gram matrix of $y_{1}, y_{2}, \ldots, y_{n}$. The determinant $g\left(y_{1}, y_{2}, \ldots, y_{n}\right)$ of the Gram matrix is known as the Gram determinant.

It is known, see for example [8], 9], that $g\left(y_{1}, y_{2}, \ldots, y_{n}\right) \geq 0$ and $g\left(y_{1}, y_{2}, \ldots, y_{n}\right)=0$ if and only if $y_{1}, y_{2}, \ldots, y_{n}$ are linearly dependent.

Proposition 2.3. Let be given the linear varieties

$$
V_{\vec{b}}:=\left\{\vec{b}+B \vec{u}: \vec{u} \in \mathbb{R}^{l_{1}}\right\}, V_{\vec{c}}:=\left\{\vec{c}-C \vec{v}: \vec{v} \in \mathbb{R}^{l_{2}}\right\},
$$

where $\vec{b}$ and $\vec{c}$ are any vectors in $\mathbb{R}^{m}$ and $B \in \mathbb{R}^{m \times l_{1}}, C \in \mathbb{R}^{m \times l_{2}}$ are fixed matrices.

Let consider $\vec{d}=\vec{c}-\vec{b}$ and assume that $A=[B C]=\left[\overrightarrow{a_{1}} \overrightarrow{a_{2}} \ldots \overrightarrow{a_{n}}\right] \in \mathbb{R}^{m \times n}, n=l_{1}+l_{2}$, is a full column rank matrix. Then:

(A) The optimal pair $\left(\vec{b}^{*}, \vec{c}^{*}\right)$ is obtained as

$$
\vec{b}^{*}=\vec{b}+B \vec{u}^{*}, \vec{c}^{*}=\vec{c}-C \vec{v}^{*},
$$

with 


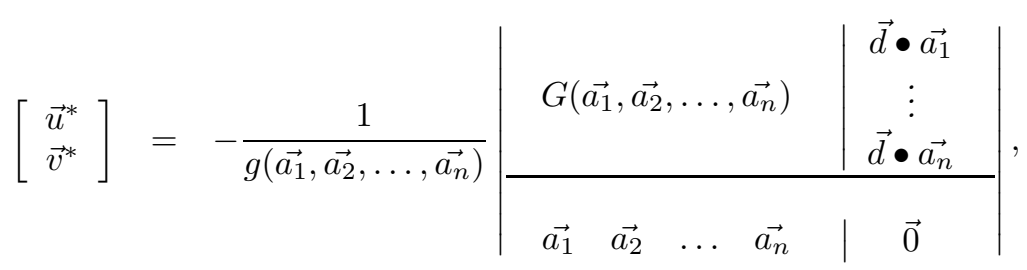

where the (formal) determinant is to be expanded by the last row to yield a linear combination of the columns $\overrightarrow{a_{1}}, \overrightarrow{a_{2}}, \ldots, \overrightarrow{a_{n}}$ of the matrix $A$.

(B) The distance between $V_{\vec{b}}$ and $V_{\vec{c}}$ is given by

$$
d\left(V_{\vec{b}}, V_{\vec{c}}\right)=\left\|\vec{b}^{*}-\vec{c}^{*}\right\|
$$

and also

$$
d^{2}\left(V_{\vec{b}}, V_{\vec{c}}\right)=\frac{g\left(\vec{d}, \overrightarrow{a_{1}}, \overrightarrow{a_{2}}, \ldots, \overrightarrow{a_{n}}\right)}{g\left(\overrightarrow{a_{1}}, \overrightarrow{a_{2}}, \ldots, \overrightarrow{a_{n}}\right)}
$$

Proof. From [3, page 196] we must minimize $\|A \vec{x}-\vec{d}\|$, where $\vec{x}=\left[\begin{array}{c}\vec{u}^{*} \\ \vec{v}^{*}\end{array}\right]$ and this is equivalent to find the distance from the point $\vec{d}$ to the column-space Range(A) of A.

By hypothesis, the columns of the matrix A are linearly independent. Then from 9 , page 74] we obtain (1) and from [4, page 65] we obtain (2).

\section{REFERENCES}

[1] A. Ben-Israel and T. N. E. Greville, Generalized Inverses: Theory and Applications, SpringerVerlag, New York, 2003.

[2] R. Caseiro, M. A. Facas Vicente and J. Vitória, Projection Method and the Distance Between Two Linear Varieties, submitted for publication.

[3] A. Dax, The Distance between Two Convex Sets, Linear Algebra and Applications, 416, 2006, pp. 184-213.

[4] F. Deutsch, Best Approximation in Inner Product Spaces, Springer, New York, 2001.

[5] A. M. DuPré and J. S. Kass, Distance and Parallelism between Flats in $\mathbb{R}^{n}$, Linear Algebra and Applications, 171, 1992, pp. 99-107.

[6] M. A. Facas Vicente, F. Martins, C. Costa, P. Tadeu and J. Vitória, Best Approximation Pair of Two Linear Varieties via an (In)Equality by (Fan-Todd) Beesack, accepted for publication.

[7] J. Gross and G. Trenkler, On Least Squares Distances between Affine Subspaces, Linear Algebra and Applications, 237/238, 1996, pp. 269-276.

[8] P.-J. Laurent, Approximation et Optimisation, Hermann, Paris, 1972.

[9] D. G. Luenberger, Optimization by Vector Space Methods, J. Wiley, New York, 1969.

[10] C. D. Meyer, Matrix Analysis and Applied Linear Algebra, SIAM Society for Industrial and Applied Mathematics, 2000. 